\title{
Optical Biosensor based on Silicon-on-Insulator Microring Cavities for Specific Protein Binding Detection
}

\author{
Katrien M. De Vos ${ }^{\mathrm{a}}$, Dr. Irene Bartolozzi ${ }^{\mathrm{b}}$, Prof. Peter Bienstman ${ }^{\mathrm{a}}$, Prof. Roel Baets ${ }^{\mathrm{a}}$, Prof. Etienne \\ Schacht $\mathrm{t}^{\mathrm{b}}$ \\ ${ }^{\mathrm{a}}$ Departement of Information Technology, Photonics Research Group, Sint-Pietersnieuwstraat 41, \\ B-9000 Gent, Belgium, mailto:Katrien.Devos@intec.ugent.be \\ ${ }^{b}$ Polymer Chemistry \& Biomaterials Research Group, Krijgslaan 281 (S4 Bis), B-9000 Gent, \\ Belgium
}

\begin{abstract}
Label-free biosensors for protein detection try to overcome the stability and reliability problems of commercialized systems relying on the detection of labeled molecules. We propose a micron sized integrated Silicon-on-Insulator optical biosensor based on a microring cavity that enables real time and sensitive measurements of protein dynamics, fast sample preparation and multiparameter detection for extremely low analyte quantities. Fabrication with Deep UV lithography for standard CMOS processing allows for cheap mass production and integration with electronic functions for complete lab-on-chip devices. The SOI material system offers a high refractive index contrast suitable for the fabrication of submicron sized optical cavities of very high quality. The shift of resonance wavelength that occurs when the dielectric surroundings of such a cavity is changed, is used for sensing. We demonstrate a SOI optical microring resonator with radius 5 micron capable of detecting bulk refractive index changes of $10^{-4}$. Modification of the semiconductor surface, allowing for immobilization of biomolecules, is characterized by X-ray Photoelectron Spectroscopy, ellipsometry, Scanning Contact Angle and Atomic Force Microscopy. We use the avidin/biotin high affinity couple to demonstrate good repeatability and the detection of protein concentrations down to $50 \mathrm{ng} / \mathrm{ml}$. Negative control using low interaction protein couples shows low responses, proving the realization of real specific binding. Integration with a microfluidic setup will allow for more precise monitoring of the interaction dynamics, while lining up the microrings in arrays will allow for cheap high throughput label-free multiparameter analyses.
\end{abstract}

Keywords: optical biosensor, label-free detection, protein interaction, microring resonator, silicon-on-insulator, avidin/biotin

\section{INTRODUCTION}

Optical label-free biosensors for protein detection attempt to overcome the drawbacks of commercialized microarrays, which rely on the detection of labeled molecules. The intermediate labeling step however complicates the detection process and decreases reliability. Direct detection of biomolecules enables the monitoring of the dynamics of molecular reactions, quantitative concentration measurements, and determination of affinity constants. When direct biosensors can be integrated on a chip, high throughput screening of biomolecular interactions in microarrays becomes possible without an intermediate labeling step. Different approaches for integrated optical biosensors based on Surface Plasmon Resonances (SPR) ${ }^{1,2,3}$, interferometers ${ }^{4,5,6}$ and resonant cavities ${ }^{7,8,9,10}$ are previously reported. Often these components still have relatively large interaction surfaces and are not suited for cheap high throughput fabrication. The integrated Silicon-on-Insulator (SOI) optical biosensor we propose enables quantitative and real time measurements, fast sample preparation, detection of extremely low analyte quantities. Fabrication with a high throughput fabrication method using standard CMOS processing, in particular deep ultraviolet lithography, allows for cheap mass fabrication and integration with electronics for real lab-on-chip devices.

Nanoscale Imaging, Spectroscopy, Sensing, and Actuation for Biomedical Applications IV, edited by Alexander N. Cartwright, Dan V. Nicolau, Proceedings of SPIE Vol. 6447, 64470K, (2007) - 1605-7422/07/\$15 - doi: 10.1117/12.698875 
SOI offers a high refractive index contrast suitable for the fabrication of nanophotonic circuits including micron- and submicron sized optical cavities of very high quality. The shift of the resonance wavelength that occurs when the dielectric surroundings of such a cavity are changed by biomolecular binding can be used for sensing. The enhanced light-matter interaction in a cavity increases the sensitivity while keeping the sensor's dimensions small. Integrated in a microfluidic setup thousands of cavities can be lined up in arrays for multiparameter sensing within a few square millimeters.

Biosensing based on semiconductor materials requires chemical modification of the silicon surface in order to provide a suitable biointerface ${ }^{11}$. Apart from being chemically and morphologically homogeneous, the surface modification both provides the immobilization of the biomolecule (ligand) in its most active conformation and prevents nonspecific protein adsorption, which can affect the sensitivity and specificity of the biosensor.

The rest of the paper is structured as follows. A description of the device and its working principle is given in paragraph 2. Paragraph 3 covers the research on the optimization and characterization of the silicon surface modification. In paragraph 4 we present measurements of the complete SOI sensor device. Its characteristics for both bulk refractive index sensing and surface sensing are given. A comparison of specific and nonspecific protein interaction is made, proving that real specific avidin/biotin binding is obtained.

\section{SOI MICRORINGS FOR SENSING PURPOSES: DESIGN AND FABRICATION}

We designed a microring cavity in an add-drop filter configuration, with one incoupling and two outcoupling ports. This is illustrated in Fig. 1 where the optical chip is mounted on a temperature controlling stage and covered by a flow cell to control liquid flows over the microring resonator, as further discussed in paragraph 4 . Whispering gallery modes occur

when incoming light with a wavelength that satisfies: $\lambda=\frac{n_{e f f} L}{m}, \mathrm{~m}=1,2, \ldots$ and $\mathrm{L}$ being the circumference, couples to the ring resonator ${ }^{12}$. This results in a sharp dip in the transmission, the spectra at the pass and the drop port are schematically shown in Fig.1.

A change of the refractive index of the ring's environment shifts the resonance spectrum, which can be monitored both by scanning the wavelength and by measuring the intensity profile at one well chosen wavelength. For both techniques the sensitivity increases with increasing quality factors $Q$ of the resonator ${ }^{13,14}$. A high Q-factor expresses narrow

spectral peaks: $Q=\frac{\Delta \lambda_{3 d B}}{\lambda_{\text {resonance }}}$.

A number of parameters determine the cavity's Q-factor. Firstly, reduced optical losses enhance the Q-factor. Secondly, a larger gap between the in- and outcoupling waveguides reduces the coupling coefficient and therefore the losses, although this reduces the throughput attenuation at the same time. Finally, smaller radii will increase bend losses on the one hand but reduce scattering losses because of decreasing round trip length on the other hand. An optimum for the radius can be found, although in our fabricated devices scattering losses are dominant, so the radius of $5 \mu \mathrm{m}$ is not the lower limit yet. Q-factors over 20,000 are achievable with our fabrication process and optimized design. The $3 \mathrm{~dB}$ peak width of the microring resonator is $75 \mathrm{pm}$.

The Silicon-On-Insulator material system highly reduces the dimensions of the devices compared to traditional passive photonic components. Thanks to the high refractive index contrast, the waveguide core is largely reduced in size to keep the waveguide single mode and total internal reflection guiding is guaranteed for very small bend radii. A consequence of the high lateral index contrast is that the waveguides become more sensitive to scattering at roughness on the corecladding interface. To this end high-quality and high-resolution fabrication tools are required. Deep ultraviolet (UV) lithography, the technology used for advanced complementary metal-oxide-semiconductor (CMOS) fabrication offers both the required resolution and the throughput needed for commercial applications. Standard CMOS fabrication 
processes were adopted to improve their capability for fabricating photonic nanostructures. This was previously reported in ${ }^{15}$.

Smaller cavity dimensions will allow sensitive detection of small amounts of analytes, since the wavelength shift will not be influenced by the microring's radius when the surface is homogenously covered. Smaller cavities will require less analyte for full coverage of the surface, this makes us believe that microcavities have a great potential for single molecule detecting biosensors. Decreasing the radius will furthermore increase the free spectral range (FSR), which will enhance the possibilities for multiarray ring configurations in future work.

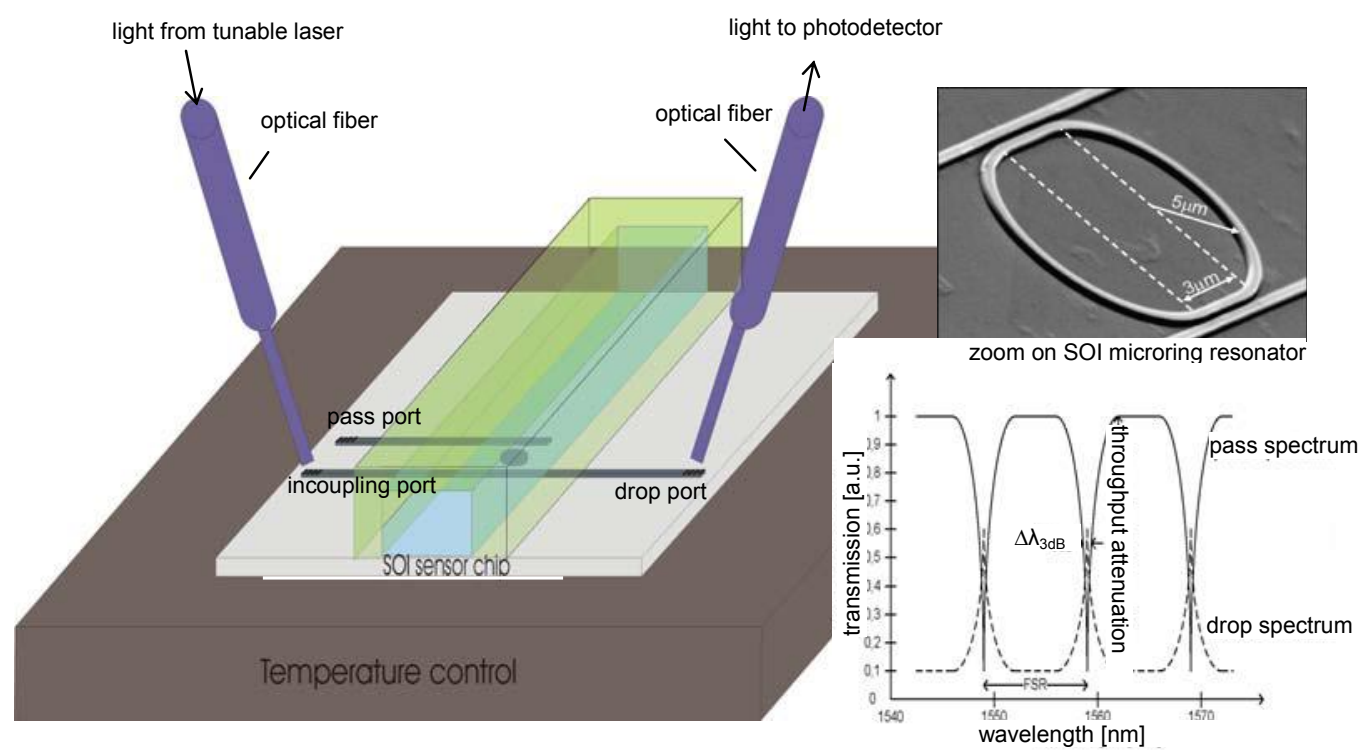

Fig. 1: Microring in add-drop filter configuration integrated in the sensing measurement setup.

\section{SILICON SURFACE FUNCTIONALIZATION}

Silanization of the surface by condensation of surface silanols with functionalized silanes can be used to provide a suitable biointerface between the transducer element and the biological medium. Silanization is commonly done by dipcoating the sample in a silane solution ${ }^{16}$. Polysiloxanes form very stable layers but unlike conventional selfassembled monolayers they have a less well-ordered structure, the $\mathrm{Si}-\mathrm{O}-\mathrm{Si}$ bond is more rigid and therefore it prevents optimal packing of the alkyl chains. The functional groups available on the polysiloxane layer can further react with a biomolecule or with a flexible, hydrophilic spacer that reduces nonspecific adsorption on the surface ${ }^{17}$. In the present work we used the avidin/biotin system, which has a high affinity constant ( $K_{a}=10^{15} M^{-1}$ ) and therefore gives a very specific and stable interaction, as a model of biomolecular ligand/receptor interaction. Biotin was immobilized on the aminofunctionalized silicon surface and the biotinylated surface was exposed to an avidin solution to allow the complex formation.

\subsection{Surface modification}

Surfaces were modified according to the following procedures. Toluene and N,N-dimethylformamide (DMF) were distilled before use. 3-aminopropylsilane (APTES) and succinimidyl-6(biotinamido)hexanoate (E,Z link-NHS-LCBiotin) were used as received.

\section{Surface cleaning and oxidation}


A preliminary oxidative step is required in order to expose hydroxyl groups on the surface. Surfaces were cleaned and oxidized in Piranha solution $\left(\mathrm{H}_{2} \mathrm{SO}_{4}: \mathrm{H}_{2} \mathrm{O}_{2}, 7: 3\right)$ at $50^{\circ} \mathrm{C}$ for $1 \mathrm{hr}$, rinsed with $\mathrm{DI}-\mathrm{H}_{2} \mathrm{O}$, dried with nitrogen and used immediately afterwards for further modifications.

\section{Silanization of silicon surfaces}

Cleaned and oxidized silicon surfaces were silanized by dip-coating at room temperature in $1 \%$ dry toluene solution of APTES. The substrates were then rinsed thoroughly with toluene and $\mathrm{DI}-\mathrm{H}_{2} \mathrm{O}$. Finally they were dried with nitrogen and stored under vacuum.

\section{Coupling of Biotin-LC-NHS}

Aminosilanized surfaces (Si-APTES) were immersed in 2ml PBS pH 7.5 and $100 \mu$ of E,Z link-NHS-LC-Biotin in DMF $(1 \mathrm{mg} / \mathrm{ml})$ was added. The reaction was carried out for 3 hours at room temperature. Surfaces were rinsed with PBS containing $0.025 \%$ Tween 20 and with $\mathrm{DI}-\mathrm{H}_{2} \mathrm{O}$ and dried with nitrogen.

\subsection{Surface characterization}

Surfaces were characterized after each modification step by static contact angle (OCA 20 from Dataphysics, distributed by Benelux Instrument), XPS (FISONS S-PROBE, provided with a fine focus Al-K source with a quartz monochromator), AFM (Nanoscoop III, Digital) and ellipsometry (Applied Materials Ellipsometer II, $\lambda=632.8 \mathrm{~nm}$ and $\alpha=70^{\circ}$ ). Measurements were carried out on flat Si surfaces. The obtained data are given in the next paragraphs.

\section{Static Contact Angle}

Contact angle was measured by monitoring a $2 \mu 1$ drop of DI water on the surface over time. After the oxidation treatment, the surface exhibited an increased hydrophilicity (contact angle decreased from $63.9^{\circ}$ to $26.2^{\circ}$ as a consequence of the exposure of the hydroxyl groups on the surface). In contrast, aminosilanization increased hydrophobicity compared to the oxidized silicon and the contact angle values increased when increasing the APTES concentration from $0.2 \%$ to $2 \%$ from about $65^{\circ}$ up to about $75^{\circ}$. This result indicates that the aminopropylsilane layer is not well ordered and part of the hydrophilic amino end-groups is interacting with the surface by hydrogen-bonding to the surface silanols with the hydrophobic aliphatic backbone exposed towards the top of the surface. By increasing the APTES concentration, multilayers are formed on the surface and the amino groups can also intramolecularly interact with the underlying layers of silanes, causing a further increase in surface hydrophobicity.

\section{X-Ray Photoelectron Spectroscopy (XPS)}

After the aminosilanization step with $0.2 \%$ APTES solution, the carbon content increased from $13.7 \%$ of residual contamination after the cleaning step to $46.2 \%$. At the same time silicon content decreases from $37.7 \%$ to $25.2 \%$. Nitrogen is absent in bare silicon, while after modification, the surface nitrogen content was about $2.2 \%$. Similar results were obtained also with the other APTES concentrations of 1 and $2 \%$. By increasing the concentration a thicker surface silane layer is obtained, that shields the bulk silicon signal, which progressively decreases to 19.9 and $17.8 \%$ respectively. On the contrary, when the APTES concentration increases to 1 or $2 \%$, the nitrogen content increases to 3.2 and 3.9\% respectively. After biotinylation only slight changes in the surface composition were detected, as expected from the small size of biotin molecules.

\section{Atomic Force Microscopy (AFM)}

AFM imaging of the bare silicon surface displayed a very low roughness of about $0.35 \mathrm{~nm}$. After the silanization, the surface displayed a fairly homogeneous morphology with globular structure at a submicron scale and a roughness ranging between $0.5-2.2 \mathrm{~nm}$. Roughness and globular nuclei size increased slightly upon increasing the concentration in the silanizing solution, suggesting the formation of multilayers and aggregation areas by the silane, as already suggested by the contact angle and XPS measurements. An overview is shown in figure 2. Biotinylated surfaces didn't show any significant morphology change detectable by AFM, as expected from the small size of the biotin molecules.

Physical and chemical characterizations confirmed the homogeneous modification of the silicon surface with aminopropylsilane but gave no direct proof of the biotin binding on the surface, which was instead confirmed by ellipsometry and measurements carried out with the sensing setup, as shown in the next paragraphs. 

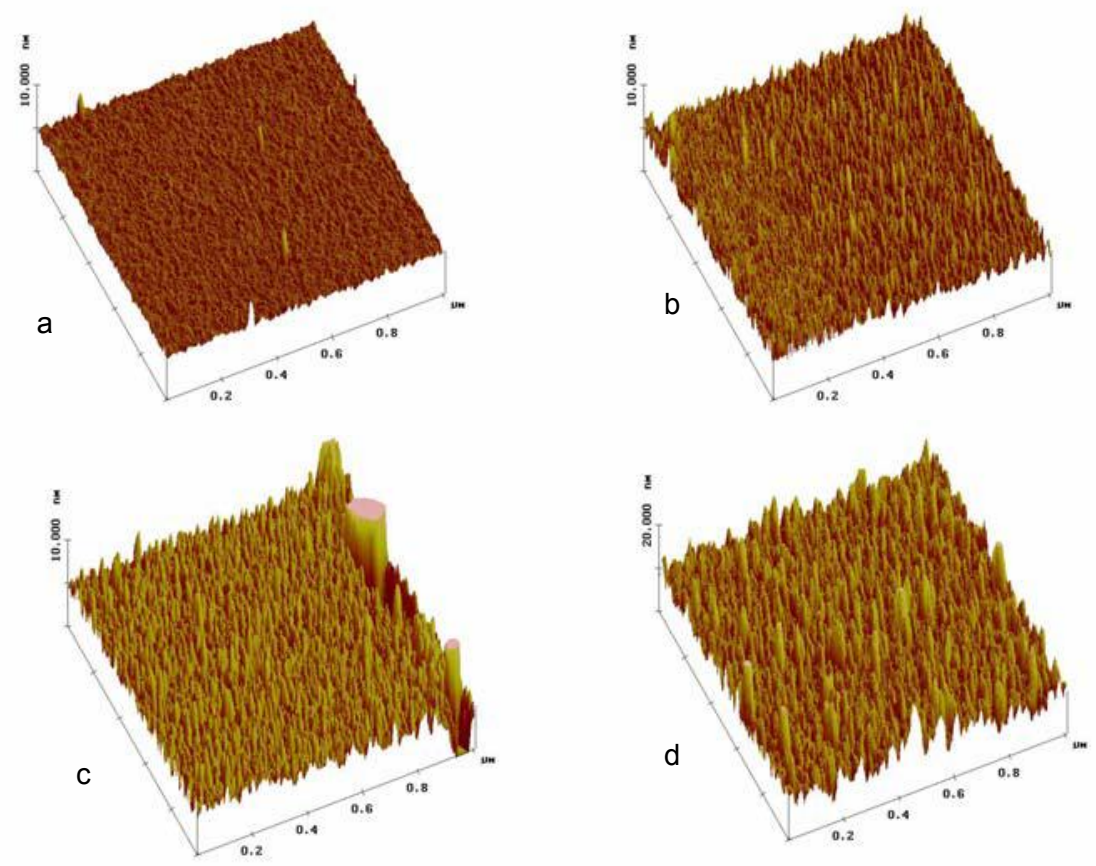

Fig. 2 : AFM images of modified samples. a) $\mathrm{Si}_{2}$, b) $\mathrm{SiA} 0.2 \%$, c) $\mathrm{SiA} 1 \%$, d) $\mathrm{SiA} 2 \%$

\section{Ellipsometry}

Ellipsometric measurements are used to determine the thickness of the layer after each modification step. The refractive index for all the surface layers is assumed to be 1.45 , in agreement with literature stated values ${ }^{18}$. After silanization with APTES, the thickness increased from $2.8 \mathrm{~nm}$ of the plain silicon surface, to $9.5 \mathrm{~nm}$ suggesting multiple layers formation during APTES deposition. Biotin immobilization did not significantly affect the thickness (about $10.1 \mathrm{~nm}$ ), but after incubation of the biotinylated surface with avidin followed by extensive rinsing, the thickness increased to $14.9 \mathrm{~nm}$, indicating the immobilization of the large avidin molecules on the surface, as consequence of the strong interaction of avidin with the biotinylated surface. On the contrary, when the aminosilanized surface - not biotinylated was exposed to avidin solution, no significant increase in thickness was observed, suggesting that no significant interaction between avidin and the surface took place. Comparing the two experiments proves the presence of biotin on the surface and therefore the effectiveness of the surface biotinylation. This is further confirmed by the biosensor measurement results, where again the response for avidin binding to both biotinylated and aminosilanized surfaces are compared (see paragraph 4.3).

\section{SENSING MEASUREMENTS}

\subsection{Measurement setup}

A schematic drawing of the proof-of-principle measurement setup is given in Fig.1. The optical chip is placed on a temperature stabilized chuck. A flow cell is mounted on the chip, connected through tubings with a peristaltic pump in order to switch easily between the liquids flown over the sensor. Measurements are done statically; we make sure we measure when all reactions have reached equilibrium, except for the real time measurement in section 4.3.

Optical fibers are vertically coupled to the in- and output integrated waveguides through grating couplers ${ }^{15}$. This allows for easy coupling with high alignment tolerances. Light coming from a tunable laser, passes the integrated circuit and is detected with a photodetector. This way a wavelength resolution of $1 \mathrm{pm}$ is obtained. 


\subsection{Bulk refractive index sensing}

Liquids with varying refractive indices (water with different sodium chloride concentrations) are flown across the ring resonator in order to characterize the sensor for bulk refractive index sensitivity. No surface chemistry was carried out, so the refractive index is homogenously changed above the ring resonator. At $20^{\circ} \mathrm{C}$ the refractive index of an aqueous solution of $\mathrm{NaCl}$ varies with 0,0018 RIU (Refractive Index Units) per mass \% ${ }^{19}$. Fig.3 shows a linear shift of the resonance wavelength with increasing salt concentration of $70 \mathrm{~nm} / \mathrm{RIU}$. All measurements are done three times to prove repeatability. The linear fit is depicted with mean wavelength shift values and weighted variance error bars. The variations are very small, proving a very high refractive index sensor's stability. A shift of one tenth of the peak broadness is easily measurable, so a minimal detectable wavelength shift of $7.5 \mathrm{pm}$ corresponds with a minimal detectable refractive index shift of $1 * 10^{-4}$ RIU.

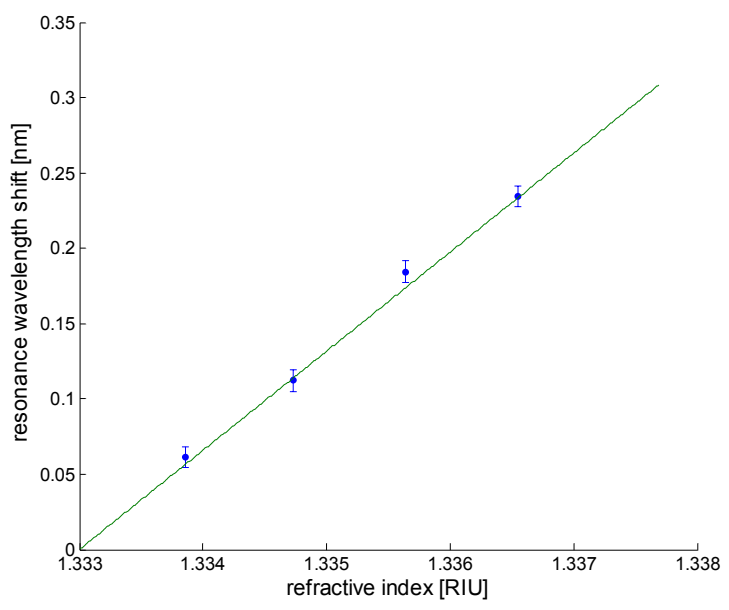

Fig. 3: Resonance wavelength shift versus bulk refractive index change.

\subsection{Surface sensing of specific protein interaction}

The high affinity protein couple avidin/biotin is used to characterize the biosensor. The silicon surfaces are modified like described in section 3, so the SOI sensor is covered with a layer of biotin molecules. We compare the resonance wavelength of the cavity immersed in Phosphate Buffer Solution (PBS), before and after being in contact with avidin solution (avidin in PBS). Redundant avidin molecules are rinsed thoroughly with PBS, so no bulk refractive index changes are involved. The evolution of the wavelength shift for different avidin concentrations compared to the reference PBS resonance wavelength is shown in Fig.4. All measurements are performed with a least 3 different samples, and error bars are indicated. For high avidin concentrations the surface is fully covered, the resonance wavelength shift saturates. This is another proof that we detect a surface modification and not a bulk liquid modification, like in figure 3 . For low avidin concentrations we can clearly see a decreased wavelength shift, the shift corresponds to the avidin concentration for quantitative molecular sensing. The estimated lowest detectable concentration, for a minimal detectable wavelength shift of $7.5 \mathrm{pm}$, is $50 \mathrm{ng} / \mathrm{ml}$. This compares well with commercially available label-free protein detection methods ${ }^{20}$.

Specificity is an important feature of a label-free biosensor. To prove that real specific avidin/biotin binding occurs, 2 nonspecific binding tests are accomplished. Firstly Bovine Serum Albumin (BSA), a protein with similar molecular weight to avidin but with low affinity to biotin, is brought into contact with the biotin layer. In graph 5 we clearly see a lower interaction. Secondly avidin is brought into contact with the aminosilanized surface without biotin, which also results in a smaller wavelength shift due to a decreased interaction of avidin with the surface, as shown in graph 6 . No specific binding occurs in the last experiment, but there might still be residual adsorption due to interaction of avidin with APTES. 


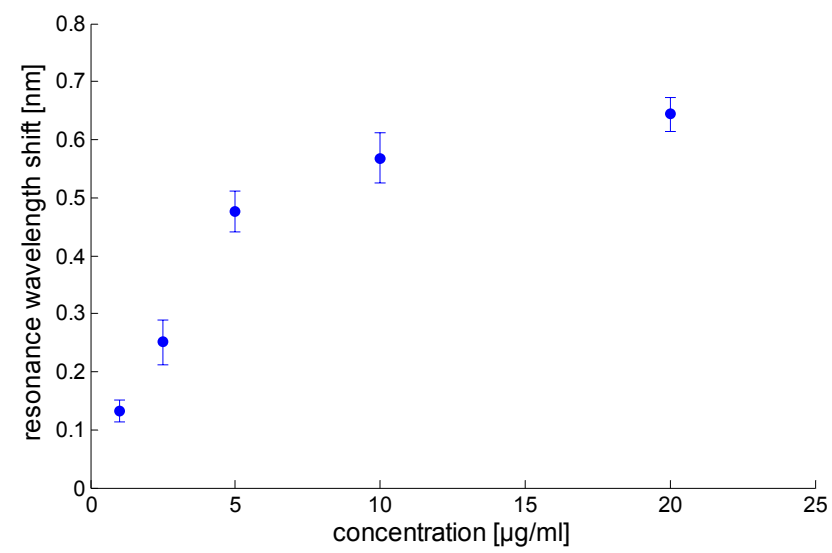

Fig. 4: Quantitative avidin-biotin detection with a SOI microring cavity.

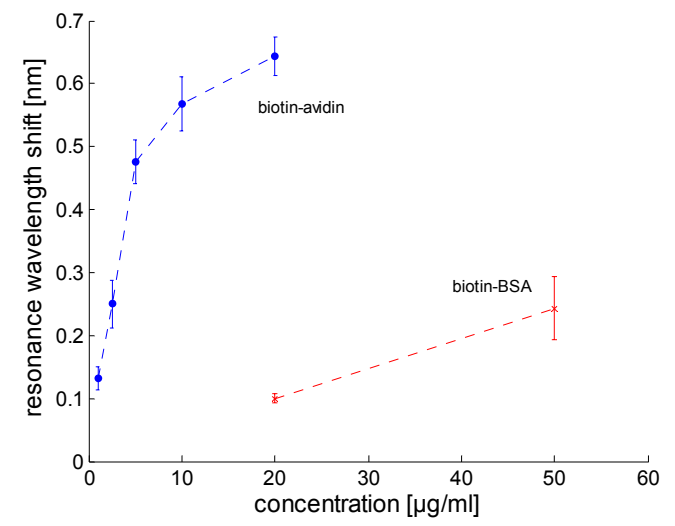

Fig. 5: Nonspecific binding tests: Avidin/BSA

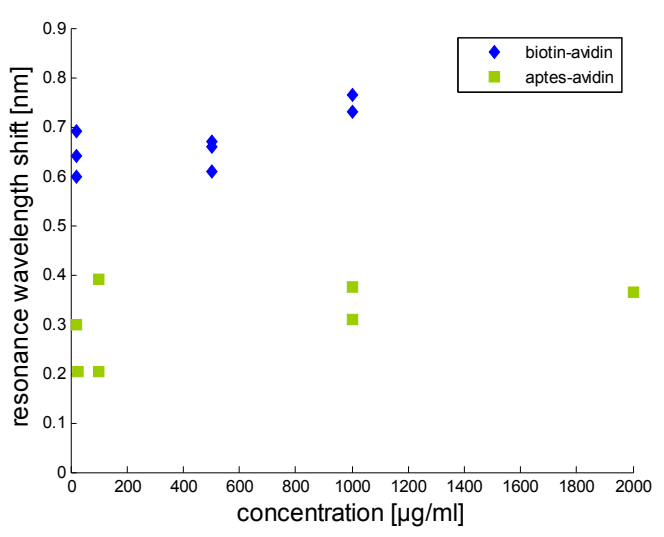

Fig. 6: Nonspecific binding tests: APTES/Avidin.

\section{CONCLUSIONS AND PERSPECTIVES}

We have demonstrated a highly miniaturized optical label-free biosensor based on a Silicon-on-Insulator microring cavity. Its high Q-factor and its extreme small size allow this device to sense low concentrations of analyte. The mass fabrication method by deep-UV lithography makes the devices suitable for high throughput biosensing in commercial applications. Measurements reveal proper operation of the device, being able to detect protein concentrations down to $50 \mathrm{ng} / \mathrm{ml}$ for the large avidin molecule, which compares favorably with commercial biosensing applications. A decreased wavelength shift for low affinity protein couples shows that the surface modification of the silicon surface succeeded in being specific for the avidin/biotin couple.

Silicon surface was modified for biosensing purposes in a two step reaction consisting in aminosilanization and biotin covalent binding. Chemical characterization by XPS and AFM confirmed the success of the homogeneous modification. Contact angle and ellipsometry suggested the formation of silane multilayers on the surface and the partial interaction of the hydrophilic amino functional groups with the silanols of the surface or of the underlying silane layers. However, coupling reaction of biotin on the aminosilanized surface was effective to provide a biotinylated surface which was used in binding experiments with avidin. 
Further improvements will be made to increase the sensitivity, by increasing the cavity's Q-factor and reduce its size. Efforts will be done to reduce nonspecific binding with antifouling molecules and a microfluidic setup will provide flexible liquid delivering with a continuous flow rate. Microrings will be lined up in arrays to perform multiarray molecular detection.

\section{ACKNOWLEDGEMENTS}

This work was carried out in the context of GOA project B/05958/01 (Ghent University). K. De Vos thanks the Flemish Institute for the Promotion of Innovation through Science and Technology (IWT) for a specialization grant and Luc Haentjes for the technical support. P. Bienstman acknowledges the Flemish Fund for Scientific Research (FWOVlaanderen) for a postdoctoral fellowship.

\section{REFERENCES}

1. Čtyrocký J., Homola J., Lambeck P.V., Musa S., Hoekstra H.J.W.M., Harris R.D., Wilkinson J.S., Usievich B., Lyndin N.M., "A Surface Plasmon Resonance Based Integrated Optical Sensor”, Sens. Actuators B, 54, 66-73, 1999.

2. Homola J., "Present and Future of Surface Plasmon Resonance Biosensors", Anal. Bioanal. Chem., 377, 528-539, 2003.

3. Lukosz W., "Principles and Sensitivities of Integrated Optical and Surface Plasmon Sensors for Direct Affinity Sensing and Immunosensing", Biosensors \& Bioelectronics, 6, 215-225, 1991.

4. Brandenburg, A., "Differential refractometry by an integrated-optical Young interferometer", Sens. Actuators B, 3839, 266-271, 1997.

5. Hradetzky D., Mueller C., Reinecke H., "Interferometric Label-free Biomolecular Detection System”, J. Opt. A: Pure Appl. Opt., 8, S360-S364, 2006.

6. Ymeti A., Kanger J.S., Greve J., Besselink G.A.J., Lambeck P.V., Wijn R., Heideman R.G., "Integration of Microfluidics with a Four-channel Integrated Optical Young Interferometer Immunosensor", Biosensors and Bioelectronics, 20, 1417-1421, 2005.

7. Boyd R.W., Heebner J.E.,. "Sensitive Disk Resonator Photonic Biosensor”, Applied Optics, 40, 5742-5747, 2001.

8. Chao C-Y., Fung W., Guo L.J., "Polymer Microring Resonators for Biochemical Sensing Applications", IEEE J. Sel. Topics Quantum Electron., 12, 134-142, 2006.

9. Ksendzov A., Lin Y., "Integrated Optics Ring-resonator Sensors for Protein Detection”, Opt. Lett., 30, 3344-3346, 2005.

10. Yalçin A. Popat K.C., Aldridge J.C., Desai T.A. Hryniewicz J., Chbouki N., Little B.E., King O., Van V., Chu S., Gill D., Anthes-Washburn M., Ünlü M.S., Goldberg B.B., "Optical Sensing of Biomolecules Using Microring Resonators", IEEE J. Sel. Topics Quantum Electron., 12, 148-155, 2006.

11. Orth R.N., Clark T.G., Craighead H.G., "Avidin-Biotin Micropatterning Methods for Biosensor Applications", Biomedical Microdevices, 5, 29-34, 2003.

12. Schwelb O., "Transmission, Group Delay and Dispersion in Single-Ring Optical Resonators and Add/Drop Filters A Tutorial Overview", J. Lightwave Technology, 22(5),1380-1394, 2004.

13. Chao C-Y., Guo L.J., "Design and Optimization of Microring Resonators in Biochemical Sensing Applications", J Lightwave Technology, 24, 1395-1402, 2006.

14. Krioukov E., Greve J., Otto C., "Performance of Integrated Optical Microcavities for Refractive Index and Fluorescence Sensing", Sensors and Actuators B, 90, 58-67, 2003.

15. Bogaerts W., Baets R., Dumon P., Wiaux V., Beckx S., Taillaert D., Luyssaert B., Van Campenhout J., Bienstman P., Van Tourhout D., "Nanophotonic Waveguides in Silicon-on-Insulator Fabricated with CMOS Technology", J. Lightwave Technology, 23, 401-412, 2005.

16. Zhang F., Srinivasan M.P., "Self-Assembly Molecular Films of Aminosilanes and their Immobilization Capacities", Langmuir, 20, 2309-2314, 2004.

17. Schreiber F., "Self-Assembled Monolayers: from 'Simple' Model Systems to Biofunctionalized Interfaces", J. Phys.: Condens. Matter, 16, R881-R900, 2004.

18. Vörös J., "The Density and Refractive Index of Adsorbing Protein Layers", Biophysical Journal, 87, 553-561, 2004.

19. Lyde D.R., Handbook of Chemistry and Physics, 77th ed., CRC press, London, 1997-1998.

20. www.biacore.com, application note 48 . 